\title{
Nivel de congestionamiento del tráfico vehicular de la zona comercial de la avenida Bolognesi, Tacna - 2019
}

\author{
Congestioning level of vehicle traffic of the commercial zone of the avenue \\ Bolognesi, Tacna - 2019
}

\author{
${ }^{1}$ Avelino Godofredo Pari Pinto \\ ${ }^{2}$ Víctor Malpartida Arrieta \\ ${ }^{3}$ Helard Olave Colque
}

\author{
ORCID: 0000-0002-6321-0721 \\ ORCID: 0000-0002-9638-9940
}

\begin{abstract}
RESUMEN
El crecimiento poblacional de una ciudad genera un crecimiento del parque automotor y, a falta de una política de ordenamiento del sistema de transporte, zonas con mayor nivel de congestionamiento. Dicha situación sucede en la ciudad de Tacna. La avenida Bolognesi es un tramo altamente comercial que comprende una vía de subida y otra de bajada, divididas por una plataforma peatonal intermedia, donde se visualizan las famosas palmeras que caracterizan a la ciudad. Ante ello, la presente investigación tuvo como objetivo determinar el nivel de congestionamiento de las vías relacionadas con dicha zona comercial. En total se estudiaron 11 puntos en los turnos de mañana, tarde y noche, durante las denominadas "horas punta", aplicando la metodología usada por Gardilci \& otros (2014). Asimismo, se realizó una encuesta a transeúntes y conductores que transitaban por la zona para conocer el efecto del nivel de congestionamiento. Los resultados fueron los siguientes: En el turno mañana, 8 obtuvieron un nivel alto, 2 obtuvieron un nivel medio y 1 obtuvo un nivel bajo. En el turno tarde, 6 alcanzaron un alto nivel y 5 alcanzaron un nivel medio. En el turno noche, 7 obtuvieron un alto nivel y 4 alcanzaron un nivel medio. De las encuestas se recogió la información de malestar por parte de los conductores, quienes afirmaron perder tiempo y dinero. Por ello, se concluye que, generalmente en algunos puntos de la zona, el nivel de congestionamiento es alto, lo que conlleva al bloqueo de las líneas de cruce peatonal, así como el bloqueo de la demarcación de "no bloquear intersecciones", especialmente en las intersecciones que no llevan dispositivos de control de tránsito.
\end{abstract}

Palabras clave: Congestionamiento, dispositivos de control de tránsito, parque automotor, nivel de congestionamiento, reglas de tránsito, tránsito.

\section{ABSTRACT}

The population growth of a city generates a growth of the automotive fleet and, in the absence of a transport system management policy, areas with a higher level of congestion. This situation happens in the city of Tacna. Bolognesi Avenue is a highly commercial stretch that includes a way up and down, divided by an intermediate pedestrian platform, where the famous palm trees that characterize the city are displayed. Given this, the objective of this investigation was to determine the level of congestion of the roads related to this commercial area. In total, 11 points were studied in the morning, afternoon and night shifts, during the socalled "peak hours", applying the methodology used by Gardilci \& others (2014). Likewise, a survey was carried out on passers-by and drivers who were passing through the area, to know the effect of the level of congestion. The results were as follows: In the morning shift, 8 obtained a high level, 2 obtained a medium level and 1 obtained a low level. In the late turn, 6 reached high level and 5 reached a medium level. On the night shift, 7 obtained a high level and 4 reached a medium level. From the surveys, the information of discomfort was collected by the drivers, who claimed to waste time and money. Therefore, it is concluded that, generally in some parts of the area, the level of congestion is high, which leads to the blockage of the pedestrian crossing lines, as well as the blocking of the demarcation of "do not block intersections", especially in intersections that do not carry traffic control devices.

Keywords: Automotive park, congestion, level of congestion, traffic, traffic rules, traffic control devices.

\footnotetext{
${ }^{1}$ Escuela Profesional de Ingeniería Mecánica. Universidad Nacional Jorge Basadre Grohmann. Tacna - Perú. E-mail: avelinopari@yahoo.es ${ }^{2}$ Escuela Profesional de Ingeniería Mecánica. Universidad Nacional Jorge Basadre Grohmann. Tacna - Perú. E-mail: vmalpartidaj@hotmail.com ${ }^{3}$ Escuela Profesional de Ingeniería Mecánica. Universidad Nacional Jorge Basadre Grohmann. Tacna - Perú. E-mail: ajavs2015@gmail.com
} 


\section{INTRODUCCIÓN}

E1 descuido por parte de las autoridades gubernamentales de generar un desarrollo equitativo de las ciudades ha hecho que las ciudades capitales se conviertan en principales centros de actividades económicas y la población tienda a desplazarse a estas. Dichas actividades generan necesidades de transporte, tanto de personas como de mercancías. Pero lamentablemente, el transporte genera diversos impactos ambientales adversos, tales como: congestión vehicular, contaminación del aire, ruido e invasión de la tranquilidad, accidentes de tránsito, etc. (Lozano, 2003).

De acuerdo al diccionario de la Lengua Española (Real Academia Española, 2001) la palabra congestión se define como "acción y efecto de congestionar o congestionarse", en tanto que "congestionar" significa "obstruir o entorpecer el paso, la circulación o el movimiento de algo", que en nuestro caso es el tránsito vehicular o llamado también tráfico vehicular.

Según Bayona (2016), la congestión se considera una externalidad negativa porque la acción de un conductor afecta a otros conductores, pero este costo no es pagado por el conductor que lo genera. Es decir, la externalidad ocasiona que el costo privado de un viaje sea diferente al costo social. El costo privado es el costo en que incurre el conductor y el costo social es el costo privado más el costo externo. El tiempo de viaje dependerá del flujo vehicular. Por ello, uno de las causas del congestionamiento es la falta de planificación, es decir no se toma en cuenta: el desarrollo poblacional, diseño geométrico vial y aplicación de métodos de transporte (Rodríguez, 2015).

La congestión del tráfico en entornos urbanos y metropolitanos perturba la eficiencia de la logística de la ciudad contemplada como "fábrica de movilidad", afectando a todos los colectivos sociales de forma directa e indirecta, empeorando la calidad de vida y penalizando las actividades comerciales y el acceso y entrega de productos en "la última milla" (Campos, 2012).

Thonsom, en su investigación realizada el 2012, representa esquemáticamente el concepto de congestión de tránsito. A medida que aumenta el tránsito, se reducen cada vez más fuertemente las velocidades de circulación. El gráfico mostrado en la Figura 1 presenta, mediante la función $t=$ $f(q)$, el tiempo $(\mathrm{t})$ necesario para transitar por una calle, a diferentes volúmenes de tránsito (q) o también llamado flujo vehicular. La otra curva, $\delta$ (qt) $/ \delta q=t+q f^{\prime}(q)$, se deriva de la anterior. La diferencia entre ambas curvas representa, para cualquier volumen de tránsito (q), el aumento del tiempo de viaje de los demás vehículos que están circulando, a causa de la introducción del vehículo adicional.

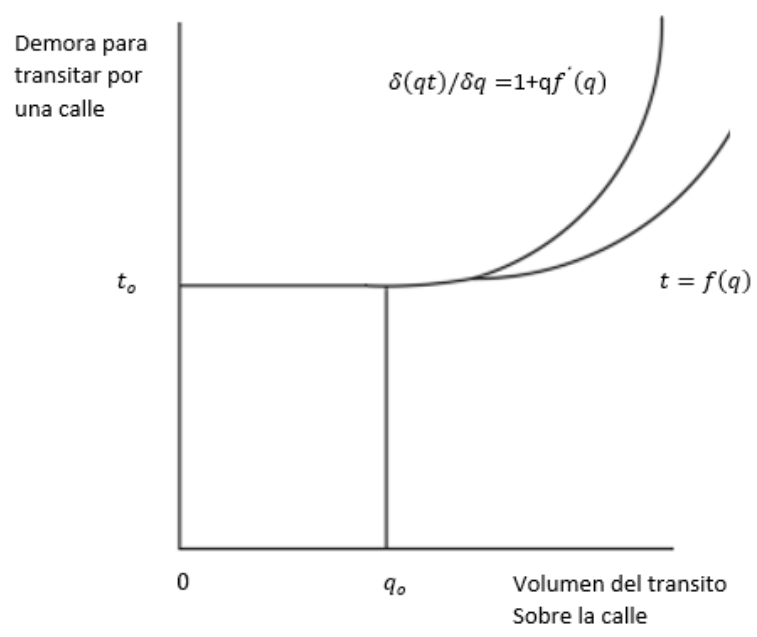

Figura 1. Representación esquemática del concepto de congestión de tránsito

Fuente: Thomson, 2012

Un análisis más técnico del tema de congestión se da en la investigación realizada por Oyola (2016), donde, para determinar la congestión de una vía, se realizaron conteos manuales de vehículos de doce horas diarias durante tres días, para obtener un volumen de dirección de recorrido. Estos datos fueron procesaros posteriormente por el método analítico HCM 1985; luego, mediante modelamiento en el programa SYNCHRO 8.0, se determinó un nivel de congestionamiento severo.

Para dar solución a la congestión vehicular, se han desarrollado una serie de teorías y propuestas planteadas en diferentes trabajos, como los modelos y métodos de optimización del tráfico urbano (Lema, 2011). Otras soluciones al tema de la congestión indican, por ejemplo, la instalación de semáforos, así como la ampliación de hasta 6 carriles (vía Pajonal, Machala Ecuador) (Oyola y otros, 2016).

Es sabido que el transporte en el Perú es netamente automotor. Y el automóvil es considerado una maquina con seis grados de libertad. Por ello, el automóvil en la actualidad ha convertido a la ciudad en un ámbito sumamente peligroso. En cada esquina que cruza el peatón 
pone en juego su vida. Además, debe soportar el aire tóxico y el ruido molesto de los automóviles (Kreimer, 2006)

La ciudad de Tacna, hoy en día, ha crecido de manera considerable y la afluencia de nuestros vecinos de Chile ha hecho que la zona comercial de una de las avenidas más populares de la ciudad, la Av. Bolognesi, sea concurrida no solamente por personas sino por vehículos, generando congestión vehicular. Al menos a partir de los años 90, el Parque Automotor de Tacna gradualmente se ha visto renovado (Pari, 2010), aunque en la actualidad por las noches circulan minibuses que realmente están fuera de servicio por obsoletos.

Muchos han sido los esfuerzos propuestos por la gerencia de Transportes de la Municipalidad de Tacna para contrarrestar la congestión vehicular de la zona comercial. Una de las soluciones consistió en dividir el carril de subida de la avenida Bolognesi en dos carriles con la ayuda de delineadores de tránsito conocidos como postes refractivos (10 de julio del 2015). De tal manera que, por la división izquierda, circulen solo vehículos livianos incluyendo taxis; y por la división derecha circulen solo los vehículos denominados "combis" y minibuses pertenecientes al Transporte Masivo Urbano, una especie de carril para "Solo Buses". Esto se caracteriza por su bajo costo de implantación, pero su rendimiento, excepto el caso a contraflujo, depende de la voluntad de cumplimiento de la medida por parte de los automovilistas (Bull, 2003). Con tal medida, se solucionó un problema, pero se derivaron otros que se mencionan a continuación:

a) Saturación de vehículos livianos en el carril izquierdo, a tal extremo de formar una línea continua a lo largo de la avenida, lo cual crea emisión simultanea de gases tóxicos al exterior, además de contaminación ruidosa por las bocinas vehiculares. Y, a su vez, crea tiempos perdidos a taxistas y conductores particulares nacionales y extranjeros.

b) Captación irregular de pasajeros en pleno carril izquierdo, originándose actitudes informales y riesgosas por los conductores y pasajeros.

c) Contaminación visual por parte de la saturación de vehículos, teniendo en cuenta que es una zona muy transitada por gran número de turistas de procedencia extranjera.

d) Cruce de peatones de manera informal a lo largo del intervalo vial, arriesgando en cierto porcentaje su integridad física.

e) Bloqueo del área de demarcación "No bloquear intersecciones", que consiste en una malla ortogonal de color amarillo y que tiene por finalidad prohibir al conductor detener el vehículo en dicha área de intersección (Viceministerio de Transporte, 2016).

f) Bloqueo del área de cruce peatonal, que es un conjunto de líneas paralelas, cuya finalidad es indicar el lugar de cruce o paso peatonal ( Ministerio de Transporte y Comunicaciones).

Posteriormente, ante la evidencia de los problemas antes mencionados, se construyó demarcaciones de cruce peatonal en ambos lados, de subida y de bajada con una longitud aproximada de $14 \mathrm{~m}$, similar a cómo se muestra en la Figura 2.

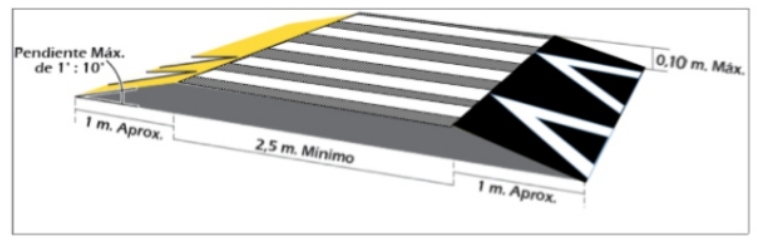

Figura 2. Vista de demarcación de cruce peatonal

Fuente: Manual del MTC, edición 2016.

Pero, la solución es mínima, por lo que los problemas ya mencionados persisten.

La presente investigación pretende analizar la problemática, determinando los niveles de congestionamiento en diferentes puntos aledaños a la zona comercial y presentar propuestas de solución a corto y largo plazo.

\section{MATERIAL Y MÉTODOS}

Los materiales utilizados fueron: tableros de apuntes, lapiceros, cronómetros para la medición del tiempo, hojas de papel bond, etc. La metodología aplicada en la presente investigación fue la metodología usada por Gardilcic y otros (2014) en la ciudad de Sucre, Bolivia. Esto consiste en determinar la calidad del tráfico vehicular de la zona, calculando el índice de congestionamiento $I c$, mediante la expresión:

$$
I_{C}=\frac{\text { Unidad de tiempo }}{N^{\circ} \text { de Vehiculos }} \text {, donde: }
$$

- Unidad de tiempo: Se refiere al tiempo de recolección de información que se emplea para contar el número de vehículos que circular por la esquina de una vía.

- $N^{\circ}$ de Vehículos: Es el número de vehículos que circularon en la unidad de tiempo.

Para obtener la unidad de tiempo, según la metodología, nos basamos en un tiempo determinado bajo el principio de obtener una 
muestra significativa de vehículos en un tiempo determinado. Ello se consigue iterando hasta llegar al tercer semáforo en rojo, tal como se muestra en la tabla siguiente:

Tabla 1. Iteración hasta llegar al tercer rojo

$\begin{array}{lclcll}\text { SEMÁFORO } & \text { [s] } & \text { SEMÁFORO } & \text { [s] } & \text { SEMÁFORO } & \text { [s] } \\ \text { Rojo } & 43 & \text { Rojo } & 43 & \text { Rojo } & 43 \\ \text { Ámbar } & 6 & \text { Ámbar } & 6 & & \\ \text { Verde } & 40 & \text { Verde } & 40 & & \\ \text { SUBTOTAL } & 89 & \text { SUBTOTAL } & 89 & \text { SUBTOTAL } & 43\end{array}$

Entonces de la Tabla 1:

Unidad de Tiempo $=89+89+43=221 \mathrm{~s}=3 \mathrm{~min}$ y $41 \mathrm{~s}=3,683 \mathrm{~min}$

Para el conteo de vehículos, se determinaron 11 puntos en toda la zona de estudio, cuyo detalle se muestra en la Tabla 2, y su ubicación se visualiza en la Figura 4.

Tabla 2. Puntos de conteo del número de vehículos

\begin{tabular}{|c|c|}
\hline Pto $N^{\circ}$ & DETALLE DEL PUNTO \\
\hline 1 & $\begin{array}{l}\text { Av. Bolognesi, altura Pallardeli. } \\
\text { Esquina farmacia (vía de subida) - } \\
\text { Carril izquierdo }\end{array}$ \\
\hline 2 & $\begin{array}{l}\text { Av. Bolognesi, altura Pallardeli. } \\
\text { Esquina farmacia (vía de subida) - } \\
\text { Carril derecho }\end{array}$ \\
\hline 3 & $\begin{array}{l}\text { Calle Pallardeli, altura Av. Bolognesi. } \\
\text { Sentido: una sola dirección. }\end{array}$ \\
\hline 4 & $\begin{array}{l}\text { Av. Bolognesi, altura Pallardeli. } \\
\text { Esquina Mercado Central }\end{array}$ \\
\hline 5 & $\begin{array}{l}\text { Av. Bolognesi, altura Av. Patricio } \\
\text { Meléndez. Esquina restaurant }\end{array}$ \\
\hline 6 & $\begin{array}{l}\text { Av. Patricio Meléndez altura Av. } \\
\text { Bolognesi. } \\
\text { Sentido: una sola dirección. }\end{array}$ \\
\hline 7 & $\begin{array}{l}\text { Av. Bolognesi, altura Av. Patricio } \\
\text { Meléndez. Esquina farmacia }\end{array}$ \\
\hline 8 & $\begin{array}{l}\text { Calle Mariscal Miller altura Av. } \\
\text { Bolognesi, vía de bajada }\end{array}$ \\
\hline 9 & $\begin{array}{l}\text { Av. Bolognesi vía de bajada con calle } \\
\text { Mariscal Miller }\end{array}$ \\
\hline 10 & $\begin{array}{l}\text { Av. Bolognesi vía de subida con calle } \\
\text { Mariscal Miller }\end{array}$ \\
\hline 11 & $\begin{array}{l}\text { Calle Mariscal Miller altura Av. } \\
\text { Bolognesi, vía de subida }\end{array}$ \\
\hline
\end{tabular}

Para el conteo se capacitaron estudiantes de la Escuela Profesional de Ingeniería Mecánica de la Universidad Nacional Jorge Basadre Grohman, para que realicen el conteo de vehículos en forma simultánea en los 11 puntos, en 3 turnos:

- Turno mañana: de 07:30 a 08:30 horas.

- Turno tarde: de 12:00 a 13:00 horas.

- Turno noche: de18:30 a 19:30 horas.

Para determinar la calidad del tráfico vehicular de la zona de estudio, determinamos los niveles de congestionamiento: bajo, medio y alto. Para ello, se tuvo en cuenta los valores siguientes:

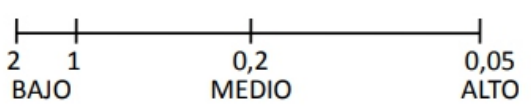

Figura 3. Valores de congestionamiento. Fuente: Gardilci \& otros, 2014

- Resultados muy próximos al valor 2 o 1 significan menores niveles de congestionamiento.

- Resultados entre 0.4 y 0.2 significan niveles de congestionamiento medio.

- Y resultados entre 0.2 y 0.05 presentan congestionamiento alto.

Por lo que, resultados mucho menores a 0.05 representan altos niveles de congestionamiento vehicular.

En la Figura 4 se muestra la ubicación exacta de los 11 puntos de análisis. Asimismo, se visualiza la dirección del flujo vehicular en cada vía. 


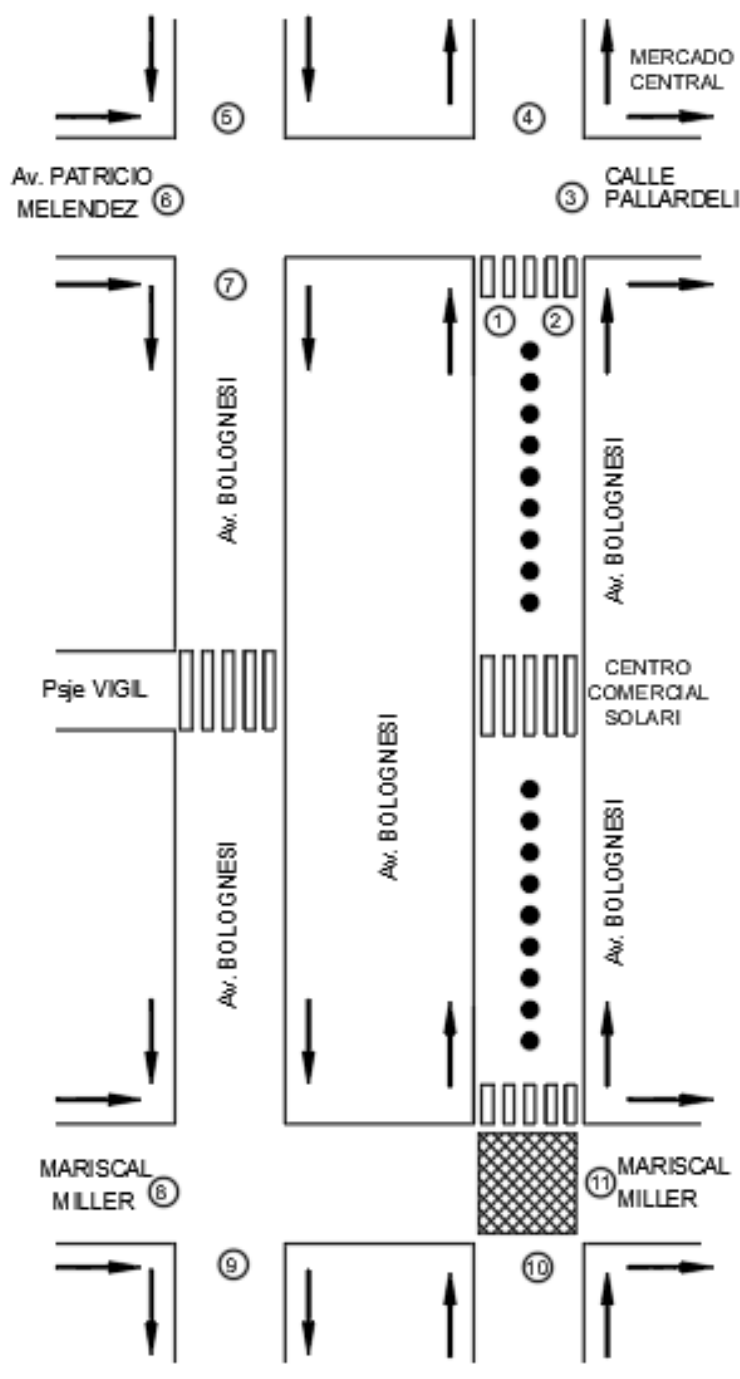

Figura 4. Vista de los 11 puntos del conteo de vehículos.

\section{RESULTADOS}

Aplicando la metodología indicada, los resultados del índice de congestionamiento en los 11 puntos de los tres turnos, indicando el número de vehículos contados, se muestran en la tabla siguiente:

Tabla 3. Resultados del Índice de Congestionamiento

\begin{tabular}{ccrcrrr} 
Pto & \multicolumn{2}{c}{ Mañana } & \multicolumn{2}{c}{ Tarde } & \multicolumn{2}{c}{ Noche } \\
s & Veh & Ic & Veh & Ic & Veh & Ic \\
& $\cdot$ & & $\cdot$ & & $\cdot$ & \\
1 & 60 & 0.06 & 34 & 0.10 & 28 & 0.13 \\
& & 1 & & 8 & & 2 \\
2 & & 0.19 & & 0.23 & & 0.26 \\
& 19 & 4 & 16 & 0 & & 3
\end{tabular}

$\begin{array}{llrlrlr}3 & & 0.06 & & 0.06 & 55 & 0.06 \\ & 54 & 8 & 56 & 6 & & 7 \\ 4 & & 0.04 & & 0.06 & & 0.05 \\ & 91 & 0 & 59 & 2 & 71 & 2 \\ 5 & & 0.08 & & 0.10 & & 0.07 \\ & 46 & 0 & 36 & 2 & 47 & 8 \\ 6 & & 0.04 & & 0.04 & & 0.03 \\ & 91 & 0 & 83 & 4 & 103 & 6 \\ 7 & & 0.07 & & 0.05 & & 0.05 \\ & 50 & 4 & 66 & 6 & 67 & 5 \\ 8 & & 0.36 & & 0.23 & & 0.28 \\ & 10 & 8 & 16 & 0 & 13 & 3 \\ 9 & & 0.09 & & 0.06 & 58 & 0.06 \\ & 41 & 0 & 58 & 4 & & 4 \\ 10 & & 0.09 & & 0.06 & 40 & 0.09 \\ & 38 & 7 & 55 & 7 & & 2 \\ 11 & & 0.73 & & 0.33 & 21 & 0.17 \\ & 5 & 7 & 11 & 5 & & 5\end{array}$

Por lo que el nivel de congestionamiento (alto, medio y bajo) en los puntos de estudio de la zona se muestra en la tabla siguiente:

Tabla 4. Nivel de congestionamiento de los puntos de la zona de investigación

\begin{tabular}{|c|c|c|c|c|c|l|}
\hline \multirow{2}{*}{ Pto } & \multicolumn{2}{|c|}{$\begin{array}{r}\text { TURNO } \\
\text { MANANA }\end{array}$} & \multicolumn{2}{c|}{$\begin{array}{c}\text { TURNO } \\
\text { TARDE }\end{array}$} & \multicolumn{2}{|c|}{$\begin{array}{c}\text { TURNO } \\
\text { NOCHE }\end{array}$} \\
\cline { 2 - 7 } & Ic & NIVEL & Ic & NIVEL & Ic & NIVEL \\
\hline 1 & 0.061 & Alto & 0.108 & Medio & 0.132 & Medio \\
\hline 2 & 0.194 & Medio & 0.230 & Medio & 0.263 & Medio \\
\hline 3 & 0.068 & Alto & 0.066 & Alto & 0.067 & Alto \\
\hline 4 & 0.040 & Alto & 0.062 & Alto & 0.052 & Alto \\
\hline 5 & 0.080 & Alto & 0.102 & Medio & 0.078 & Alto \\
\hline 6 & 0.040 & Alto & 0.044 & Alto & 0.036 & Alto \\
\hline 7 & 0.074 & Alto & 0.056 & Alto & 0.055 & Alto \\
\hline 8 & 0.368 & Medio & 0.230 & Medio & 0.283 & Medio \\
\hline 9 & 0.090 & Alto & 0.064 & Alto & 0.064 & Alto \\
\hline 10 & 0.097 & Alto & 0.067 & Alto & 0.092 & Alto \\
\hline 11 & 0.737 & Bajo & 0.335 & Medio & 0.175 & Medio \\
\hline
\end{tabular}

Nivel de congestionamiento alto

Nivel de congestionamiento medio

Nivel de congestionamiento bajo 
inferior de la zona se visualiza solo dos puntos de alto nivel y otros de nivel intermedio.

Asimismo, en la Figura 7 se visualiza 5 puntos con alto nivel de congestionamiento y con tan solo dos puntos de nivel intermedio en la parte superior. En la parte inferior los niveles de congestionamiento se presentan de la misma manera que en el turno tarde.

Observando los tres turnos, se visualiza solo un punto con nivel de congestionamiento bajo. Ello indica que la zona está congestionada en los tres turnos, por lo que el malestar de los transeúntes es prácticamente durante la jornada laboral.

\section{Turno noche:}

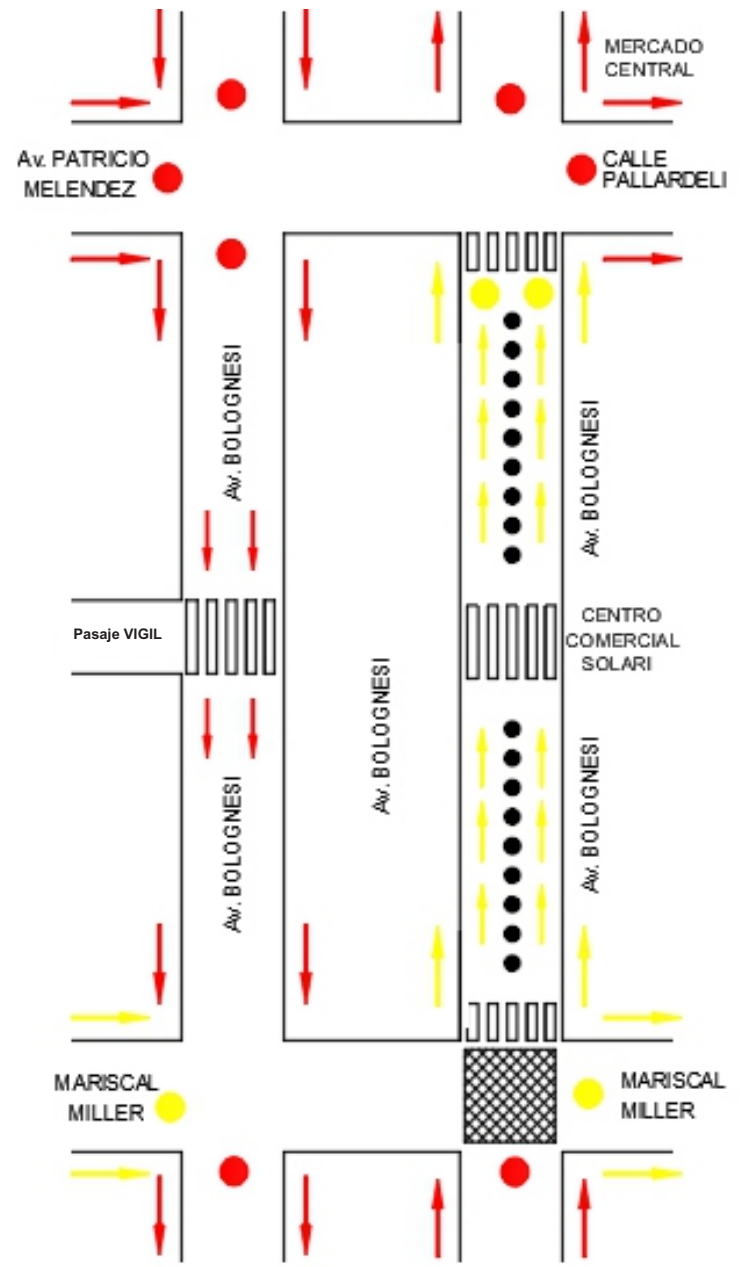

Figura 8. Puntos de congestionamiento "Turno noche"

Para corroborar el efecto de la congestión vehicular en la zona, tal como se mencionó al inicio, se aplicaron encuestas tanto a los conductores como a los transeúntes. Esto al inicio del proyecto, durante el proyecto y al finalizar el proyecto. Es decir, los resultados presentados en las tablas serán el promedio de las tres encuestas. Los resultados de las 3 encuestas se presentan en las Tablas 5 y 6 siguientes:

Tabla 5. Resultados de encuestas a transeúntes

\begin{tabular}{|c|c|c|}
\hline \multirow[b]{2}{*}{ PREGUNTAS } & \multicolumn{2}{|c|}{ RESPUESTAS } \\
\hline & $\underset{[\%]}{\text { Sí }}$ & $\begin{array}{l}\text { NO } \\
{[\%]}\end{array}$ \\
\hline $\begin{array}{l}\text { ¿Se observa congestión vehicular } \\
\text { en la zona? }\end{array}$ & 97 & 3 \\
\hline $\begin{array}{l}\text { ¿Cree que se debería tomar } \\
\text { medidas para erradicar la } \\
\text { congestión vehicular de la vía de } \\
\text { subida dividida? }\end{array}$ & 95.1 & 4.9 \\
\hline
\end{tabular}

De la Tabla N ${ }^{\circ} 5$, el $97 \%$ observa congestión vehicular en la zona de estudio y el $95.1 \%$ manifiesta que se debería tomar medidas para erradicar la congestión, especialmente en la vía de subida dividida.

Tabla 6. Resultados de encuestas a conductores

\begin{tabular}{|c|c|c|}
\hline \multirow[b]{2}{*}{ PREGUNTAS } & \multicolumn{2}{|c|}{ RESPUESTAS } \\
\hline & $\begin{array}{c}\text { SÍ } \\
{[\%]}\end{array}$ & $\begin{array}{l}\text { NO } \\
{[\%]}\end{array}$ \\
\hline $\begin{array}{l}\text { ¿Se observa congestión } \\
\text { vehicular en la zona? }\end{array}$ & 97.7 & 2.3 \\
\hline $\begin{array}{l}\text { ¿Le perjudica a usted la } \\
\text { congestión vehicular? }\end{array}$ & 96.5 & 3.5 \\
\hline $\begin{array}{l}\text { ¿Está usted de acuerdo con las } \\
\text { medidas que se han tomado } \\
\text { hasta el momento en la vía por } \\
\text { parte de la Municipalidad? }\end{array}$ & 41.7 & 58.3 \\
\hline $\begin{array}{l}\text { ¿Cree usted que se debería } \\
\text { tomar medidas más estrictas } \\
\text { para erradicar la congestión } \\
\text { vehicular de la vía de subida } \\
\text { dividida? }\end{array}$ & 93.3 & 6.7 \\
\hline $\begin{array}{l}\text { ¿Estaría usted de acuerdo con } \\
\text { pagar por circular por una vía } \\
\text { de congestión vehicular? }\end{array}$ & 18.7 & 81.3 \\
\hline
\end{tabular}

Con respecto a la pregunta: ¿De qué manera se ve usted perjudicado por la congestión vehicular?, realizada a los conductores de taxis, se tienen los siguientes resultados: 
Se presentan gráficamente los niveles de congestionamiento, según los turnos correspondientes y en los puntos de estudio de la zona en los turnos.

\section{Turno mañana:}

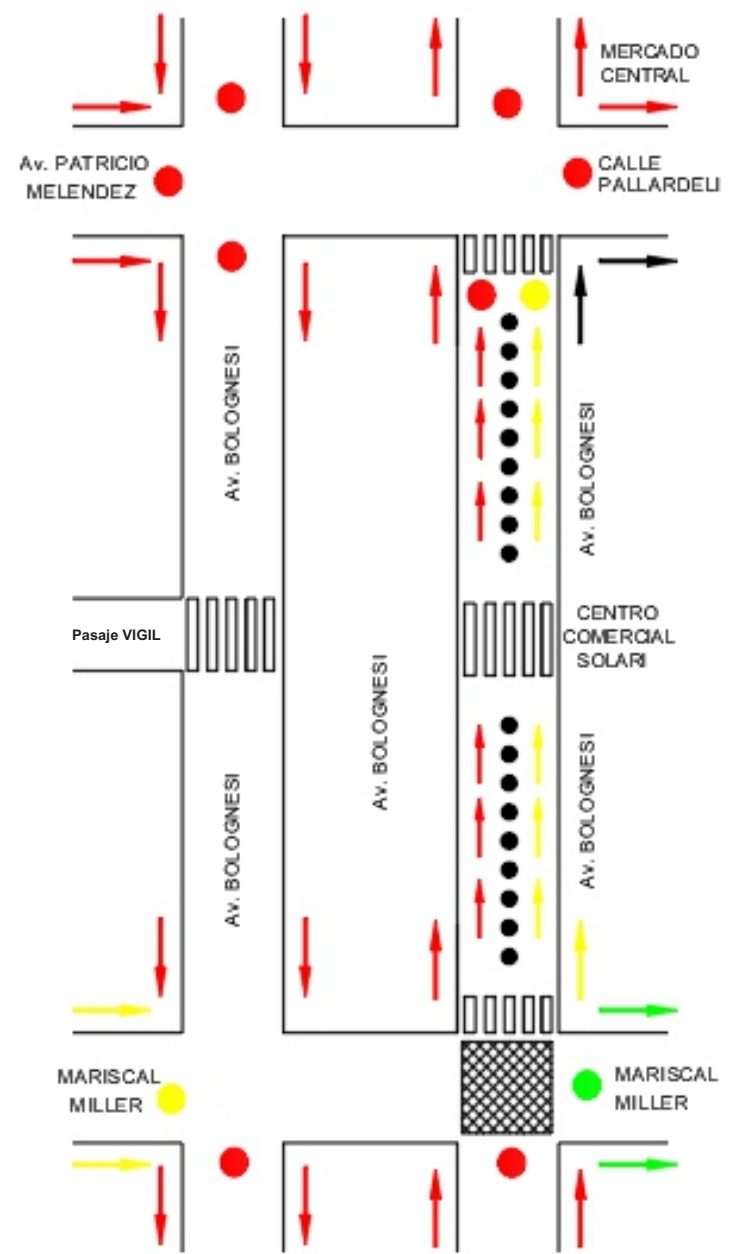

Figura 5. Puntos de congestionamiento "Turno mañana"

En la Figura 5 se observa que, en el turno de mañana, la parte más congestionada es la parte superior de la zona, con 6 puntos de alto nivel de congestionamiento y la parte inferior solo con dos. Asimismo, en el turno mañana se da solo un punto a la altura de la calle Miller con bajo grado de congestionamiento.

Es importante mencionar que en la Figura 5 se muestra el carril izquierdo de la vía de subida dividida, un alto índice de congestionamiento y es justamente este tramo materia de la presente investigación que provoca que en la intersección Miller con Avenida Bolognesi, se bloquee las líneas peatonales y la demarcación "No bloquear intersecciones".
Se corrobora el índice de congestionamiento del carril en la figura siguiente:

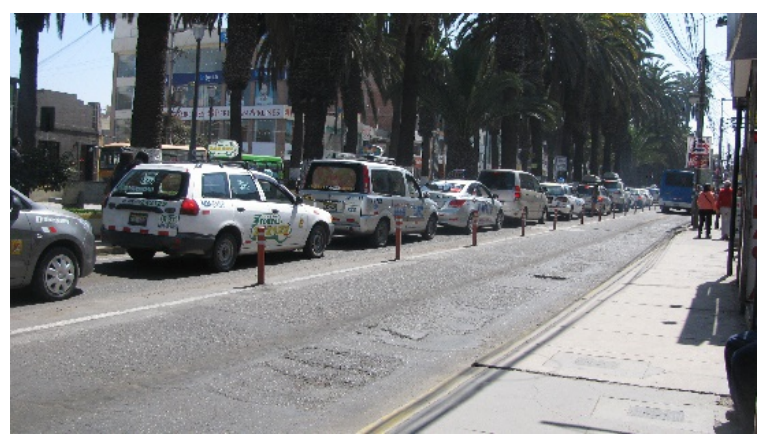

Figura 6. Carril izquierdo congestionado

\section{Turno tarde:}

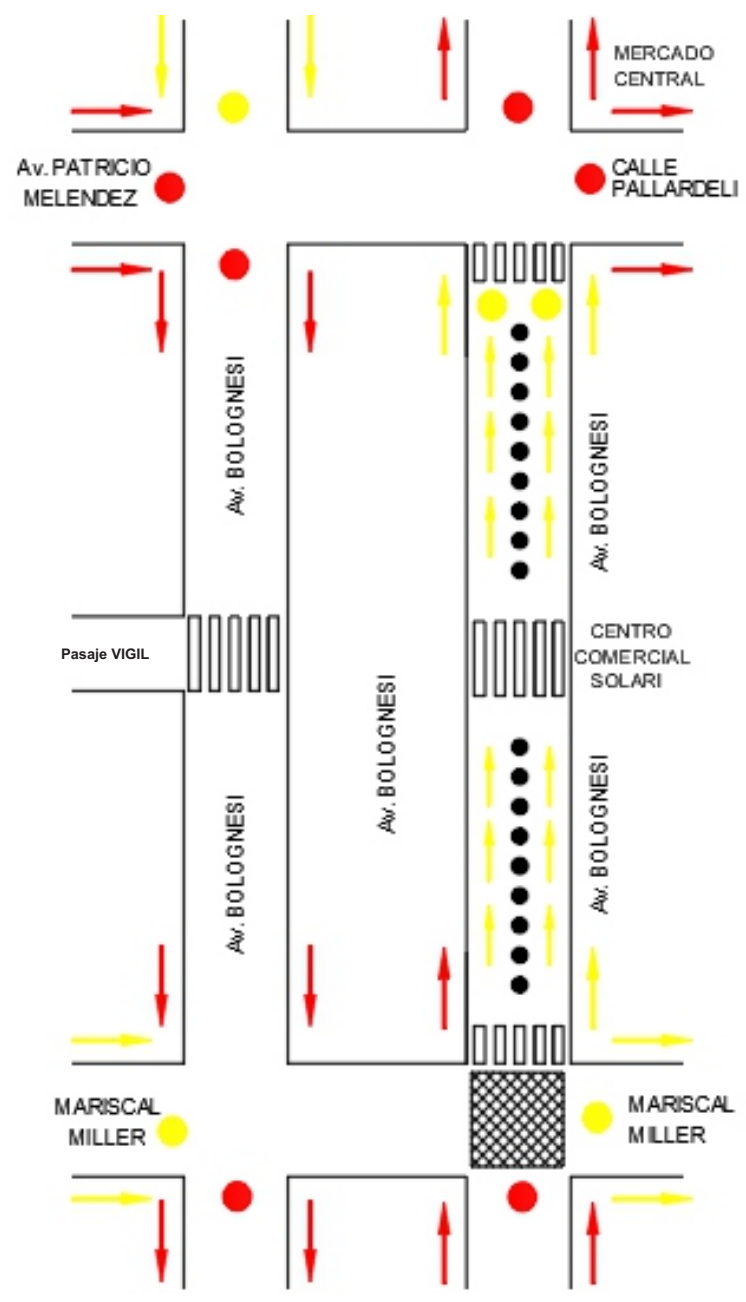

Figura 7. Puntos de congestionamiento "Turno tarde"

En la Figura 6 se visualiza en la parte superior solo 4 puntos de alto nivel de congestionamiento y 3 niveles intermedios, mientras que, en la parte 


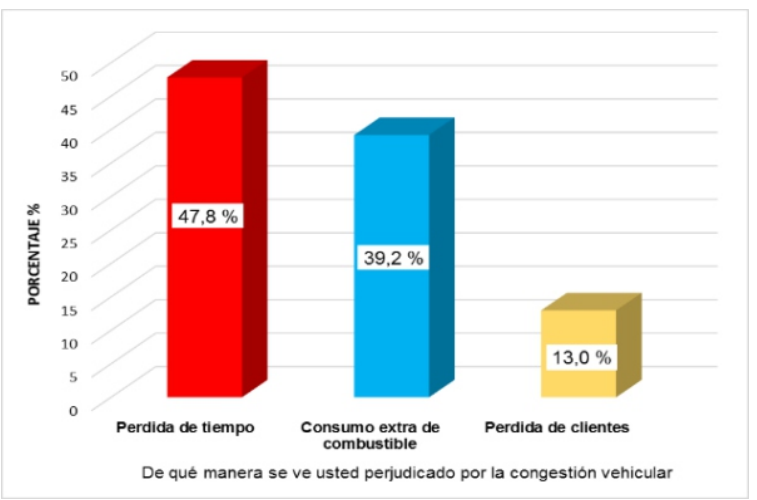

Figura 9. Resultado de preguntas a conductores de taxi

\section{DISCUSIÓN}

Se encontraron puntos con un alto nivel de congestionamiento, especialmente en la parte superior de la zona de estudio. Por tal motivo, la parte inferior donde no se cuenta con dispositivos de control de tránsito queda totalmente saturada por vehículos, bloqueando las demarcaciones: de cruce peatonal y de no bloquear intersecciones. Situación que incomoda a transeúntes.

Analizando los resultados de la Tabla 4, en el turno de la mañana, en la esquina que alcanza por Av. Bolognesi, altura Pallardeli, esquina farmacia (vía de subida) - carril izquierdo, presenta un indicador bajo $(I c=0.061)$, lo que nos dice que su índice de congestionamiento vehicular es cercano a alto.

Lo que no sucede con el carril derecho, que presenta un indicador equivalente a 0.194 , lo que nos dice que la vía tiene un nivel de congestionamiento medio.

Asimismo, en la misma tabla se observa que, en el turno de la mañana, los índices de congestionamiento son altos en 8 intersecciones. Especialmente en la intersección 4 (Av. Bolognesi, altura Pallardeli. Esquina Mercado Central) e intersección 7 (Av. Bolognesi, altura Av. Patricio Meléndez, esquina farmacia). En el turno de la tarde, el índice de congestionamiento alto se da en 6 intersecciones, mientras que en el turno de la noche se da en 7 intersecciones.

En la investigación de Gardilci \& otros (2014), en el sector 1, los valores más bajos son de $0.10,0.17$ y 0.18 , correspondientes a la calle Calvo. Y la calle Bolívar tiene valores de 0.11, 0.13, $0.15 \mathrm{y}$ 0.18 . En el sector 3, en la calle Ravello, el valor más bajo es de 0.08. Sin embargo, durante el turno de la mañana en el punto 6 (Av. Patricio Meléndez, altura Av. Bolognesi), en nuestra investigación se registró el valor de 0.040 , lo que nos dice que la zona comercial en estudio está más congestionada que la zona de Sucre, sector 3. Esto se debe al flujo de extranjeros provenientes del vecino país de Chile, que llegan a Tacna no solamente a hacer turismo, sino también a realizar actividades comerciales, como compra de artículos, consumo de alimentos y últimamente consumo de servicios como: atención de ópticas, atención médica especializada, etc.

Existen muchas propuestas de solución al congestionamiento. Una de ellas es la aplicación del sistema pico y placa, que consiste en la restricción de circulación de vehículos de acuerdo al número de su placa, controlada por un aplicativo móvil (Mendoza, 2014). Otro método de solución es la aplicación del sistema "movilidad urbana sostenible", que consiste en mejorar las condiciones del sistema de transporte, las cuales son aplicables especialmente para avenidas de gran flujo vehicular (Vera, 2017).

Una curiosa solución a la congestión es la aplicación de la política del garrote, que consiste en cobrar por el uso de vías congestionadas; de tal manera que los usuarios perciban el costo social de viajar por ellas y tomen decisiones de usar otras vías (Ortuzar, 2002). A ello, según nuestra investigación, el $81.3 \%$ de los conductores encuestados mencionan no estar de acuerdo con pagar por el uso de la vía congestionada, frente a un $18.7 \%$ que responde sí estar de acuerdo (Tabla $6)$.

\section{CONCLUSIONES}

La parte superior de la zona comercial de la avenida Bolognesi presenta un alto nivel de congestionamiento, lo cual genera bloqueo de demarcaciones de uso peatonal y vehicular.

Se comprobó el efecto de la congestión vehicular mediante encuestas a transeúntes y conductores. Las medidas de control realizadas por la Municipalidad de Tacna (dividir en dos carriles la vía de subida de la zona y la construcción de una demarcación peatonal de $15 \mathrm{~m}$ ) no son suficientes para resolver los problemas de congestión vehicular, la población exige medidas más estrictas.

Por lo que, se recomienda a la Gerencia de Transportes de la Municipalidad de Tacna adopte medidas de corto (aplicación de dispositivos de control en la calle Miller) y largo plazo (reorganización de la circulación de los vehículos pertenecientes al transporte masivo urbano, fuera de la avenida Bolognesi). 
Asimismo, se recomienda al área de transportes de la Municipalidad Provincial de Tacna, realice una campaña de educación de comportamiento vial a conductores del sector transporte masivo urbano (taxis, combis, coasters y minibuses).

\section{AGRADECIMIENTO}

Al Vicerrectorado de Investigación de la UNJBG de la ciudad de Tacna que, a través de su Instituto de Investigación, hizo posible el financiamiento del presente proyecto con RR $N^{\circ} 3725-2018$ UNJBG.

\section{REFERENCIAS}

BULL, Alberto. Congestión de tránsito. E1 problema y como enfrentarlo. Publicación de las Naciones Unidas. 2003.

BAYONA R. \& MARQUEZ T. La congestión vehicular en la ciudad de Piura. Instituto de investigación y promoción para el Desarrollo Universidad Nacional de Piura. 2015.

CAMPOS C. \& ROBUSTE A. Medida de la congestión del tráfico en ciudades. Departamento de Infraestructuras y Territorio Universidad Politécnica de Catalunia. España. 2012.

GARDILCIC, Mirko et al. Análisis de la problemática del tráfico y vialidad del centro histórico de la ciudad de Sucre. Universidad Mayor Real y Pontifica de San Francisco Xavier de Chuquisaca. Sucre Bolivia. 2014.

KREIMER, Roxana. La tiranía del automóvil. Los costos humanos del desarrollo de la tecnología. Buenos Aires. Argentina. 2006.

LEMA F. et al. Estudio de la optimización del tráfico en un cruce a través del ajuste de los ciclos de los semáforos mediante recocido simulado. XVII Jornada ASEPUMA - V Encuentro Internacional. 2011.
LOZANO A., Torres V. \& Antun J. Tráfico vehicular en zonas urbanas. Revista Ciencias de la Universidad Nacional Autónoma de México. 2003.

MENDOZA S. \& Villacis V. Análisis y solución al congestionamiento vehicular en horas pico utilizando una aplicación móvil con GPS. Universidad Politécnica Salesiana Sede Guayaquil. Ecuador. 2014.

ORTUZAR, Juan de Dios. ¿Es posible reducir la congestión? Revista ARQ. Número 52, $\mathrm{p}$ 7 - 9. Pontifica Universidad Católica de Chile. 2002.

OYOLA E. et al. Evaluación de la congestión vehicular: Av. Castro Benítez y vía Pajonal, Machaca - Ecuador, año 2016. Universidad Técnica de Machala. Ecuador. 2017.

PARI, Avelino. Influencia del estado de los vehículos gasolinero y diésel de transporte masivo urbano en la inspección de gases contaminantes vehiculares en la ciudad de Tacna - 2009 . ESPG-UNJBG. 2010.

REALACADEMIA ESPAÑOLA. Diccionario de la lengua española. 22 - ava edición. 2001.

RODRIGUEZ R., Osiris V. \& Rodríguez E. Evaluación del congestionamiento vehicular en intersecciones viales. Departamento de Ingeniería Civil y Ambiental. 2015. Universidad Autónoma de Ciudad Juárez

THOMSON I. \& Bull A. La congestión de transito urbano: causas y consecuencias económicas y sociales, Revista de la CEPAL 76. Santiago de Chile. 2002.

VERA P. \& Zapata N. Propuesta para la solución de la congestión vehicular en la avenida Javier Prado Oeste (Entre la avenida La Molina y la calle Los Tiamos), Escuela Profesional de Ingeniería Civil. Universidad San Martín de Porres. 2017.

Viceministerio de Transporte. Manual de dispositivos de control del tránsito automotor para calles y carreteras. MTC. Lima Perú. 2017. 